\title{
A phenanthrene derived PARP inhibitor is an extra-centrosomes de-clustering agent exclusively eradicating human cancer cells
}

\author{
Asher Castiel ${ }^{4 \dagger}$, Leonid Visochek ${ }^{1 \dagger}$, Leonid Mittelman ${ }^{3}$, Françoise Dantzer ${ }^{5}$, Shai Izraeli ${ }^{2,4}$ and \\ Malka Cohen-Armon ${ }^{1 *}$
}

\begin{abstract}
Background: Cells of most human cancers have supernumerary centrosomes. To enable an accurate chromosome segregation and cell division, these cells developed a yet unresolved molecular mechanism, clustering their extra centrosomes at two poles, thereby mimicking mitosis in normal cells. Failure of this bipolar centrosome clustering causes multipolar spindle structures and aberrant chromosomes segregation that prevent normal cell division and lead to 'mitotic catastrophe cell death'.
\end{abstract}

Methods: We used cell biology and biochemical methods, including flow cytometry, immunocytochemistry and live confocal imaging.

Results: We identified a phenanthrene derived PARP inhibitor, known for its activity in neuroprotection under stress conditions, which exclusively eradicated multi-centrosomal human cancer cells (mammary, colon, lung, pancreas, ovarian) while acting as extra-centrosomes de-clustering agent in mitosis. Normal human proliferating cells (endothelial, epithelial and mesenchymal cells) were not impaired. Despite acting as PARP inhibitor, the cytotoxic activity of this molecule in cancer cells was not attributed to PARP inhibition alone.

Conclusion: We identified a water soluble phenanthridine that exclusively targets the unique dependence of most human cancer cells on their supernumerary centrosomes bi-polar clustering for their survival. This paves the way for a new selective cancer-targeting therapy, efficient in a wide range of human cancers.

\section{Background}

We have recently reported the selective eradication of human triple negative mammary cancer cells MDA-231 by phenanthrene derivatives (also acting as potent inhibitors of polyADP-ribose polymerases) [1]. These compounds included the phenanthridines PJ-34 and Phen and the isoquiniline Tiq-A. They were originally designed to protect neuronal cells in the central nervous system from cell death evoked by high activity of PARP1 in response to DNA damage caused by brain injury, stroke or inflammation $[2,3]$. We found that these molecules cause G2/M transition arrest in the cell cycle of

\footnotetext{
*Correspondence: marmon@post.tau.ac.il

+ Contributed equally

${ }^{1}$ The Neufeld Cardiac Research Institute, Department of Physiology and Pharmacology, Sackler Faculty of Medicine, Tel-Aviv University, 69978, TelAviv, Israel

Full list of author information is available at the end of the article
}

both mammary cancer cells MDA-231 and normal epithelial cells MCF-10. However, while G2/M arrest was permanent in the cancer cells, and was accompanied by their massive cell death, normal mammary epithelial cells overcame the cell cycle arrest and continued to proliferate normally in the presence of these phenanthrene derivatives [1]. The most potent compound was PJ-34, which also efficiently prevented the development of MDA-231 xenotransplants in nude mice without inducing detectable toxic effects in the animals [1].

The current results outline a mechanism that apparently underlies the exclusive cytotoxicity of PJ-34 in these human mammary cancer cells. We found that this molecule acts as a centrosomes de-clustering agent in cells with supernumerary centrosomes, which are most abundant in these mammary cancer cells and in most human cancers $[4,5]$.

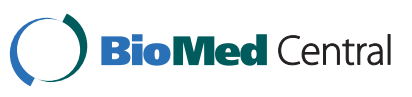

() 2011 Castiel et al; licensee BioMed Central Ltd. This is an Open Access article distributed under the terms of the Creative Commons Attribution License (http://creativecommons.org/licenses/by/2.0), which permits unrestricted use, distribution, and reproduction in any medium, provided the original work is properly cited. 
Bipolar centrosomes assembly during metaphase is crucial for bipolar spindle formation and accurate chromosomes segregation in cells undergoing mitosis $[6,7]$. To accomplish these tasks, cells dividing with more than two centrosomes have developed a yet unresolved molecular mechanism, clustering their extra-centrosomes at two poles $[4,5,8]$. Failure of this bipolar centrosome assembly causes multipolar spindle structures and aberrant chromosomes segregation that prevent normal cell division [5]. This may lead either to 'mitotic slippage' where cells 'slip' out of mitosis to re-enter G1 without satisfying the spindle assembly checkpoint (SAC), or to induction of 'mitotic catastrophe cell death' $[5,9,10]$ or 'anaphase catastrophy' [10]. Although cell death induced by failure to accomplish mitosis has been well documented, the underlying molecular mechanisms are still poorly understood $[5,10]$

Extra centrosomes are most abundant in cells of human solid cancers and in some of the human hematological malignancies $[4,5]$, whereas in normal somatic cells the number of centrosomes is restricted, via several control mechanisms, to two centrosomes per cell $[8,9,11]$. This difference between normal and cancer human cells raised the idea of using compounds that interfere with the bipolar clustering of extra centrosomes to achieve selective eradication of cancer cells without harming normal tissues [5,12].

Our findings confirm this hypothesis by identifying a molecule, which prevents extra centrosomes clustering in mitosis and exclusively eradicates human cancer cells with supernumerary centrosomes without impairing normal proliferating cells.

\section{Methods}

\section{Cells and cell cultures}

Human cancer cell lines included mammary triple negative (MDA-231) cells, lung (H1299), colon (DLD-1), ovarian (HeyA8), and pancreatic (Panc-1) cells. These cell-lines and the human epithelial cell line MCF-10A were supplied by ATCC, (Manassas, VA, USA). Human umbilical vein endothelial cells (Huvec) were supplied by PromoCell (Heidelberg, Germany), and human primary adipose-derived mesenchymal stroma cells were prepared from human thymus and kindly donated by Prof Jonathan Leor (Sheba Medical Center, Israel). The human cancer cell lines were chosen for our experiments as representatives of human cancer cells with high occurrence of extra-centrosomes.

Cancer cell lines were cultured in 6-well multi-dish plates (Nunc; Roskilde, Denmark) and maintained in a medium containing Dulbecco's Modified Eagle Medium (DMEM) containing 10\% horse serum, 1\% L-glutamine, and 1\% Pen-Strep-Amphotericin B (Gibco, UK). Epithelial MCF-10A cells were cultured in DMEM/F12 (Gibco,
UK) containing 6\% fetal bovine serum (FBS), 0.02\% epidermal growth factor $(100 \mu \mathrm{g} / \mathrm{ml}$; CytoLab, Rehovot, Israel), 2.8\% hydrocortisone (50 $\mu \mathrm{M}$; Sigma-Aldrich), $0.1 \%$ insulin $(10 \mathrm{mg} / \mathrm{ml}$; Sigma-Aldrich), and 1\% Pen/ Strep (Gibco, UK). Human endothelial cells, Huvec were cultured in endothelial cell growth medium (cat. \# C22011; Promocell, Heidelberg, Germany) and used within 3 passages. Human primary mesenchymal stroma cells were prepared from thymus and cultured in DMEM/F12 (Rhenium) containing 15\% fetal bovine serum (FBS). These cells were used within 5 passages.

\section{Treatments}

Cells were treated with phenanthrene-derived compounds acting as potent PARP inhibitors: PJ-34 (N-(6oxo-5,6-dihydro-phenanthridin-2-yl)-N,N-dimethyl-acetamide), Tiq-A, (4H-thieno[2,3-c]isoquinolin-5-one), and Phen, $(6(5 \mathrm{H})$-phenanthridinone). PJ-34 and Phen were purchased from Alexis Biochemicals (Enzo Life Sciences International, Inc. PA, USA), Tiq-A was purchased from Sigma-Aldrich. In addition, cells were treated with two potent PARP inhibitors that are not phenanthrene derivatives, ABT-888 (2-[(R)-2-methylpyrrolidin-2-yl]-1Hbenzimidazole-4-carboxamide) (Abbott Labs, Illinois, U. S.A) and BSI-201 (4-iodo-3-nitrobenzamide) (BiPar, San Francisco, CA, USA) (Figure 1).

Flow cytometry was performed with Beckton Dickenson machine and the FlowJo software (Tree Star, Ashland, OR). It was used to monitor changes in the ploidy levels of malignant and normal cells. At the indicated times, the cells were collected, permeabilized (75\% ethanol in double-distilled water), and stained with propidium iodide. Cell eradication and the kinetics of S-phase entry and G2/M transition were monitored by measuring the percentages of cells at the different phases. Cell counts at the selected time periods during 120 hours incubation indicated cell-cycle changes resulting from treatment with the tested compounds. Untreated cells were used as controls for each cell type at the selected time periods.

\section{Confocal microscope imaging and immunocytochemistry}

For immuno-cytochemistry, cells were grown on glass coverslips in the appropriate growth medium. After treatment, the cells were fixed with $4 \%$ paraformaldehyde (PFA; 20 minutes) and permeabilized with $0.2 \%$ Triton-X100 before blocking with $10 \%$ goat serum in PBS (details were described before [13]). The cells were immunolabeled for $\alpha$ and $\gamma$-tubulin. Spindles were labeled by antibodies directed against $\alpha$-tubulin (T9026; Sigma) and detected by secondary fluorescent antibody Dylight (green) (Jackson ImmunoResearch Cat \# 115485-166). Antibodies directed against $\gamma$-tubulin (polyclonal, T5192; Sigma) detected by secondary fluorescent 
<smiles>CN(C)CCCCNc1ccc2[nH]c(=O)c3ccccc3c2c1</smiles>

PJ-34

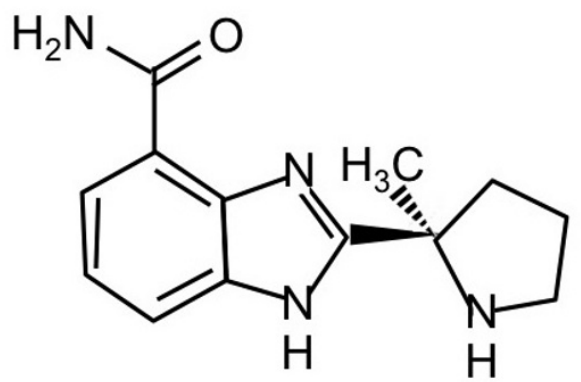

ABT-888<smiles>O=c1[nH]c2sccc2c2ccccc12</smiles><smiles>O=c1[nH]c2ccccc2c2ccccc12</smiles>

Phen

Tiq-A

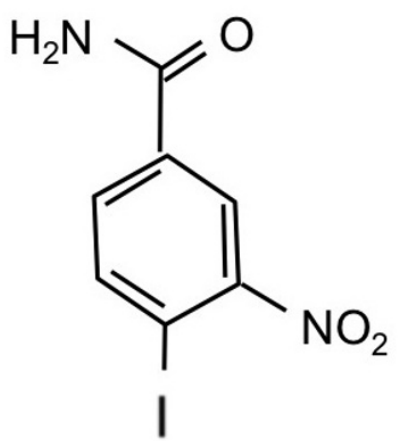

\section{BSI-201}

Figure 1 The chemical structure of the compounds tested in both cancer and benign human cells. The phenanthridines, PJ-34 and Phen, the isoquiniline Tiq-A, and the non-phenanthrene derivatives, ABT888 and BSI-201. These compounds act as potent PARP inhibitors.

antibody CyTM3 (red) were used for immunolabeling of centrosomes. In some experiments centrosomes were double labeled for $\gamma$-tubulin (monoclonal, Abcam, MA, ab11316) and for centrin1 (polyclonal, Abcam, MA, ab11257) [14]. The percentage of multi-focal spindles was calculated in most cases out of a total number of 20 spindles that were detected in different randomly selected scanned samples of each of the tested cell types.

For live confocal imaging of cells, cells were transfected using liposomal reagent (Jet-PI, Polyplus, \#101$10)$ with vectors encoding for $\gamma$-tubulin-GFP (for detection of centrosomes), $\alpha$-tubulin-RFP (for detection of spindles and microtubule arrays) and for histone H2BRED (for detection of chromosomes). Twenty-four hours after transfection the cells were exposed to PJ-34
$(20 \mu \mathrm{M})$ for 18 hours, and then incubated in the presence of PJ-34 in live imaging chamber and scanned over-night (about 15 hours) by confocal microscope imaging. All the vectors were a gift of Prof. Michael Brandeis, Hebrew University, Jerusalem.

Luminescent detection of ATP level in the cells was used for quantification of cell viability. The Cell Viability Assay Kit (Abcam, MA) enabled a rapid quantification of viable cells in the cultures (the net result of growth arrest, cell death and cell proliferation). This method is based on the correlation between cell viability and ATP production, which signals the presence of metabolically active cells [15]. High percentage of cell death, as quantified by flow cytometry, was in correlation with decreased ATP levels in the tested cell cultures. 


\section{Results}

We examined several potent PARP inhibitors, including the phenanthrene derivatives PJ-34, Tiq-A and Phen and the non-phenanthrene derivatives ABT-888 and BSI-201 (Figure 1) in human cancer cell types with a high occurrence of extra-centrosomal cells (>50\%).

In our previous experiments PJ-34 caused G2/M arrest and a massive cell death in MDA-231 mammary cancer cells without impairing normal human mammary epithelial MCF-10 cells [1]. Given that MDA-231 cells have high occurrence of extra-centrosomes, and the interference with bipolar clustering of supernumerary centrosomes causes $\mathrm{G} 2 / \mathrm{M}$ arrest $[5,16,17]$, we examined the possibility that PJ-34 affects extra-centrosomes clustering in mitosis.

Our experiments disclosed a remarkable activity of PJ34 on disruption of the bipolar clustering of supernumerary centrosomes in mammary MDA-231 cancer cells, causing distorted multi-polar spindles as early as 6 hours after PJ-34 application, followed by massive cell death within 48-72 hours (Figures 2a, b and Additional File 1). PJ-34 was applied only once to the cells, 24 hours after seeding, at twice to 3-times higher concentration than that completely inhibiting the activity of PARP-1 [3]. The cytotoxic activity of PJ-34 was identified in randomly selected fixed or live MDA-231 cells, scanned by confocal microscopy (Figures 2a, b and Additional File 1; Methods). Also, PJ-34 did not seem to impair the structure or formation of centrosomes, as indicated by centrosomes double labeling with centrin 1 and $\gamma$-tubuline (Figure 2a) and by confocal imaging of centrosomes in live MDA-231 cells in the interphase (Additional File 2).

These findings associated the remarkable cytotoxic activity of PJ-34 in MDA-231 cells with de-clustering of their extra-centrosomes in mitosis, causing multi-polar distorted spindles and aberrant chromosomes segregation that lead to 'mitotic catastrophe cell death' [5].

Other phenanthrene derived molecules acting as PARP inhibitors, Phenanthridinon (Phen), and Tiq-A (Figure 1; $[18,19]$ ), similarly interfered with centrosomes clustering in MDA-231 cells. However, their effect was milder and accompanied by a lower percentage of cell death (Figure 3).

In contrast, non-phenanthrene derivatives acting as potent PARP inhibitors, BSI-201 and ABT888 [18,19], did not share this capability. Even when used at high concentrations (2-4 times higher than concentrations completely inhibiting the activity of PARP-1) they did not impair the bifocal clustering of extra centrosomes in these cells (Figure 3).

To further test the suggested contribution of PJ-34 to extra-centrosomes de-clustering, we examined its activity in other human cancer cell-lines with high occurrence of supernumerary centrosomes.

PJ-34 similarly eradicated the tested cells, including lung (H1299), colon (DLD-1), ovarian (HeyA8), and pancreatic (Panc-1) cancer cells. PJ-34 (20-30 $\mu \mathrm{M})$ caused G2/M arrest in these cells and a subsequent massive cell death, observed 48-96 hours after PJ-34 application (Figures 4 and 5). In accordance, PJ-34 prevented the bipolar clustering of supernumerary centrosomes in these cells, causing multipolar spindles and aberrant chromosomes alignment detected within 18 hours after PJ-34 application (Figure 6). For the tested five types of cancer cell-lines, there was a significant correlation between the fraction of cells with multipolar spindles and the reduction in cell viability (as indicated by the reduction in ATP level) of the treated cells (Figures 2a, 4 and 6; $\mathrm{r}=0.92 ; \mathrm{p}<0.0001$ ). Thus, cell death could be attributed to de-clustering of their extracentrosomes.

In contrast, normal human proliferating cells were resistant to the cytotoxic activity of PJ-34, Tiq-A and Phen in human cancer cells. Even at the high concentrations used and the long incubation periods, the most potent compound, PJ-34, did not interfere with the cell cycle of benign human proliferating cells. The tested cells included human mammary epithelial cells MCF-10, mesenchymal cells prepared from human thymus and human umbilical vein endothelial cells (Huvec) (Figure 7). As for Huvec, PJ-34 did not affect their cell cycle within 48 hours incubation (Figure 7 ). These cells did not survive longer incubation periods even without treatment ( $>5 \%$ cell death was measured in untreated Huvec cells after 72 hours incubation).

In these three types of benign cells, PJ-34 did not interfere with the formation and assembly of centrosomes (two centrosomes at the poles of bifocal spindles), nor with chromosomes segregation. Bipolar assembly of centrosomes, bifocal spindle formation and chromosomes segregation were not impaired in the normal proliferating cells, even after long incubation with high concentrations of PJ-34 (Figure 8).

\section{Discussion}

Our results suggest that the exclusive eradication of human cancer cells with extra centrosomes by the phenanthridine derivative PJ-34 is attributable to its extracentrosomes de-clustering activity in mitosis (Figures 2, 4, 5, 6, Additional File 1 and Additional File 2).

According to our observations, PJ-34 did not impair centrosomes in both human normal and cancer cells, nor did it interfere with the formation of centrosomes in the interphase of multi-centrosomal cancer cells or impaired the bi-polar assembly of centrosomes in mitosis of normal proliferating cells with two centrosomes 
a

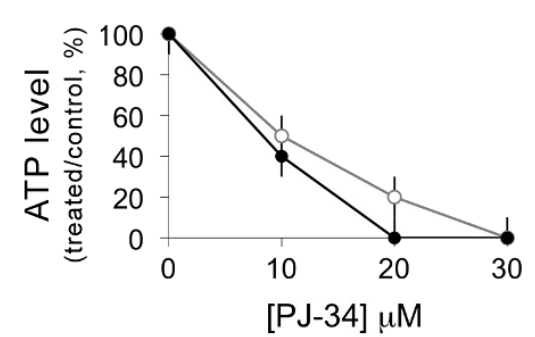

Untreated

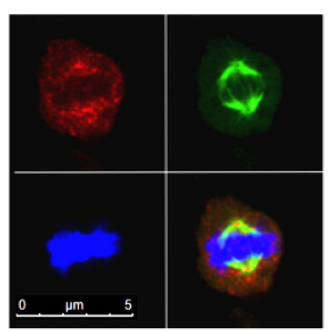

10

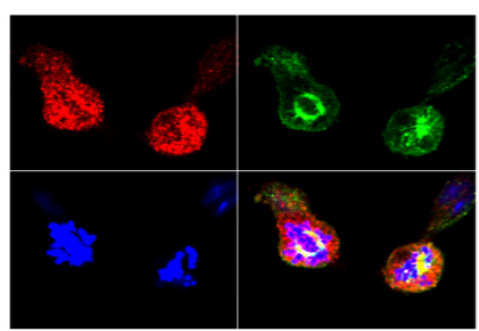

Untreated
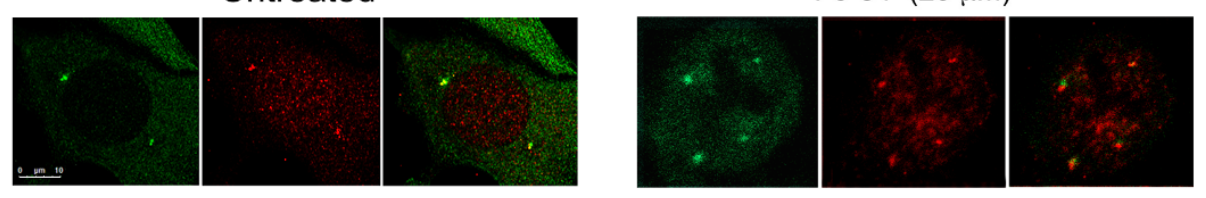

b

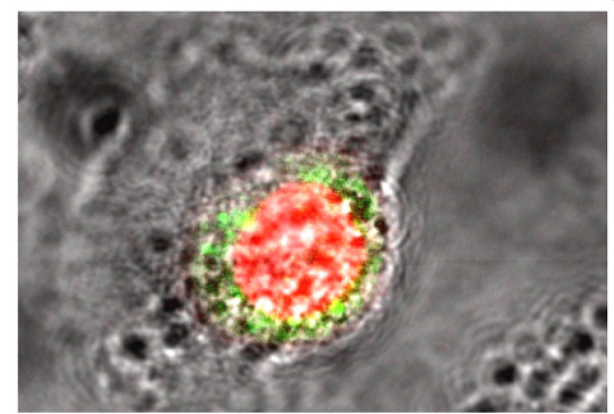

PJ-34 $(20 \mu \mathrm{M})$
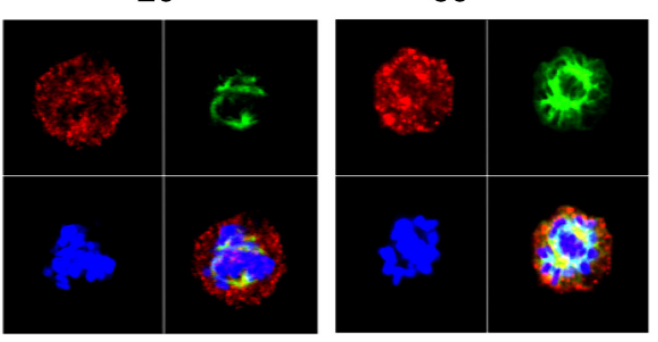

PJ-34 $(20 u \mathrm{M})$

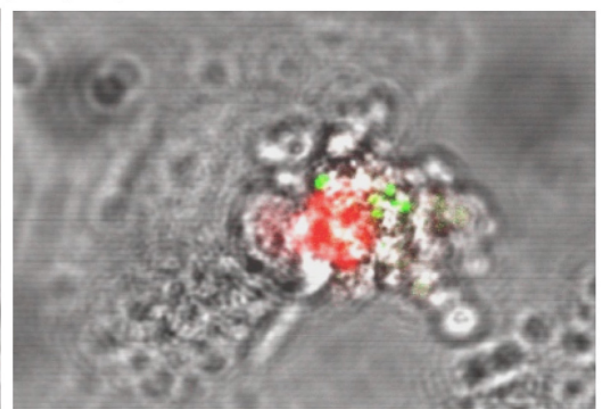

Untreated
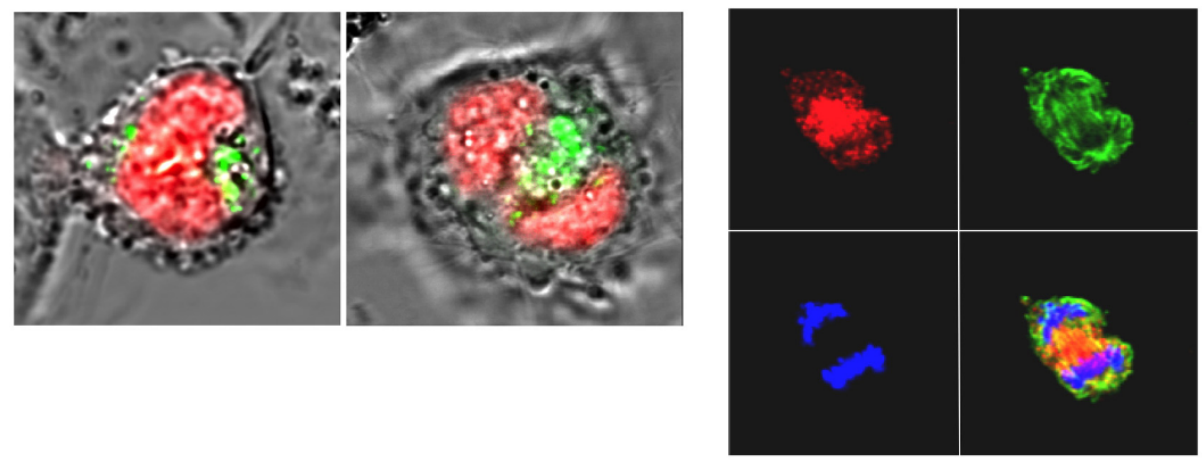

Figure 2 De-clustering of supernumerary centrosomes in mitosis preceded PJ-34 induced cell death in human mammary cancer cells MDA-231. (a) PJ-34 induced multi-polar spindles with scattered centrosomes and cell death in MDA-231 cells. Top: (Left) loss of cell viability after treatment with PJ-34 was quantified by luminescent detection of ATP levels in the treated cells (\% ATP levels in cells treated with PJ-34 relative to ATP levels of untreated, control cells) (Methods). Cells were incubated with PJ-34 (applied once at 24 hours after seeding) for 72 hours 
(grey line) and 96 hours (black line). The mean values and standard deviations of 4 measurements in 3 different experiments are presented. (Right) The percentage of distorted spindles with scattered centrosomes in cells treated with PJ-34 was calculated out of 20 detected spindles by confocal scanning of randomly selected MDA-231 cells that were incubated with PJ-34 at the indicated concentrations and durations (48 hours; grey line) and (72 hours; black line). Both untreated and treated cells were permeabilized and immunolabeled for $\alpha$ - and $\gamma$-tubulin in 3 different experiments, as explained below. Middle: Confocal images presenting scattered centrosomes and aberrant chromosomes arrangement in multifocal spindles of randomly selected MDA-231 cells that were permeabilized and immunolabeled for $\alpha$ - and $\gamma$-tubulin for the detection of spindles and centrosomes (green and red fluorescent labeling, respectively), 18 hours after application of PJ-34 at the indicated concentrations. Chromosomes were labeled with DAPI reagent (blue) (Methods). Bottom: Double immunolabeling of centrosomes for $\gamma$-tubulin (green) and centrin1 (red) in randomly selected MDA-231 cells. Bipolar clustering of 4 centrosomes in an untreated cell (Left) and 4 un-clustered centrosomes in a cell treated with PJ-34 (20 MM) (Right) (Methods). (b) Live confocal imaging indicated the effect of PJ-34 on bi-polar centrosomes' clustering in randomly selected live MDA-231 cells. MDA-231 cells were transfected with vectors encoding for histone H2b-RED (red; for chromosomes labeling) and for $\gamma$-tubulin-GFP (green; for centrosomes labeling). Randomly selected cells were scanned overnight by confocal microscopy without or during treatment with PJ-34 (Methods). Upper frame- Un-clustered centrosomes (Left) during mitosis preceded cell death (Right) in a randomly selected live MDA-231 cell that was incubated with PJ-34 $(20 \mu \mathrm{M})$ for 18 hours before scanning and during scanning (15 hours; imaging is included in Additional File 1). Lower frame- MDA-231 cells untreated with PJ-34. (Left) Live confocal images of randomly selected live MDA-231 cell with clustered centrosomes in anaphase. (Right) confocal image of randomly selected untreated fixed MDA-231 cell in anaphase. This cell was permeabilized and immunolabeled for $\alpha$-tubuline (green; spindles) and for $\gamma$-tubuline (red; centrosomes). Chromosomes were labeled with DAPI reagent (blue).

(Figures 2a, 8, Additional File 1 and Additional File 2). These features may underlie its outstanding capability to exclusively eradicate human cancer cells while keeping normal proliferating cells un-affected (Figures 5 and 7).

Theoretically, in cancer cells with high occurrence of extra-centrosomes $(>50 \%)$ when mitosis is accompanied by eradication of multi-centrosomal cells, an exponential reduction of the fraction of bi-centrosomal cancer cells is expected. In this case, the higher their proliferation rate the more rapidly will these cancer cells be eradicated by extra-centrosomes de-clustering agents.

The indicated interference of the tested phenanthrene derivatives with the bipolar clustering of supernumerary centrosomes was not shared by non-phenanthrene derivatives acting as potent PARP inhibitors as well (Figure 3). This questions an exclusive role of PARP inhibition in extra centrosomes de-clustering. Nevertheless, these results could also reflect a possible, yet un-identified selective inhibition of PARP isoforms by phenanthridines.

Results obtained from genome-wide RNAi screen in multi-centrosomal cells, including embryonic Drosophila S2 cells and human malignant cells, identified genes required to suppress multipolar mitosis. Proteins involved in the organization and regulation of the cytoskeleton, including Kinesin HSET/Ncd (which is not required for polar organization in normal somatic cells) and the four chromosomal passenger complex (CPC) components, Aurora-B, INCENP, Survivin, and Borealin were identified among the main proteins required for bifocal clustering of extra-centrosomes in these cells $[12,17]$. Evidence for the necessity of tankyrase-I and a putative human PARP-16 homolog in centrosomes clustering has been also provided $[17,20]$. PolyADP-ribosylation of tankyrase-1 may contribute to spindle bipolarity by providing a static matrix, anchoring microtubule- associated motor proteins and spindle proteins $[17,20]$. A specific role for PARP-16 in mitosis has not been identified yet [17].

PARP proteins are present in centrosomes [21-24], and both Aurora-B and INCENP are targets for polyADP-ribosylation [22,24]. In addition, supernumerary centrosomes were found in PARP-1 deficient cells [25]. Thus, in view of the exclusive interference of PJ-34 with extra centrosomes de-clustering in mitosis, a possible synergism between PARP activity and other mechanisms underlying extra-centrosomes clustering should be further investigated.

The indicated cytotoxic activity of the phenanthrene derivatives in human cancer cells with high occurrence of extra-centrosomes links for the first time between two well known, but poorly understood phenomena; Some anti-tumor effects of natural phenanthridines were first described in the early fifties [26-28], and a higher cytotoxicity of some phenanthridines in cancer cells as compared to normal cells has been reported before [29,30], although the underlying mechanisms have never been understood. In addition, cell-cycle arrest resulting from failure of centrosomes to accomplish bipolar clustering in multi-centrosomal cells has been acknowledged for nearly a century $[6,31,32]$. Nevertheless, the causal relationship between multiple centrosomes and malignancy has only recently been clarified [32-35].

Mitotic-spindle microtubules are among the most effective targets for anti-cancer therapy [5,10,17,36-39]. Therefore efforts are invested in trying to find mechanisms exclusively targeting mitosis in cancer cells [40-42].

Unlike the exclusive interference of PJ-34 with extra centrosomes bi-polar clustering in mitosis (Figures 2, 3, 4, 5, 6, Additional File 1 and Additional File 2), the currently known small molecules interfering with mitosis in 


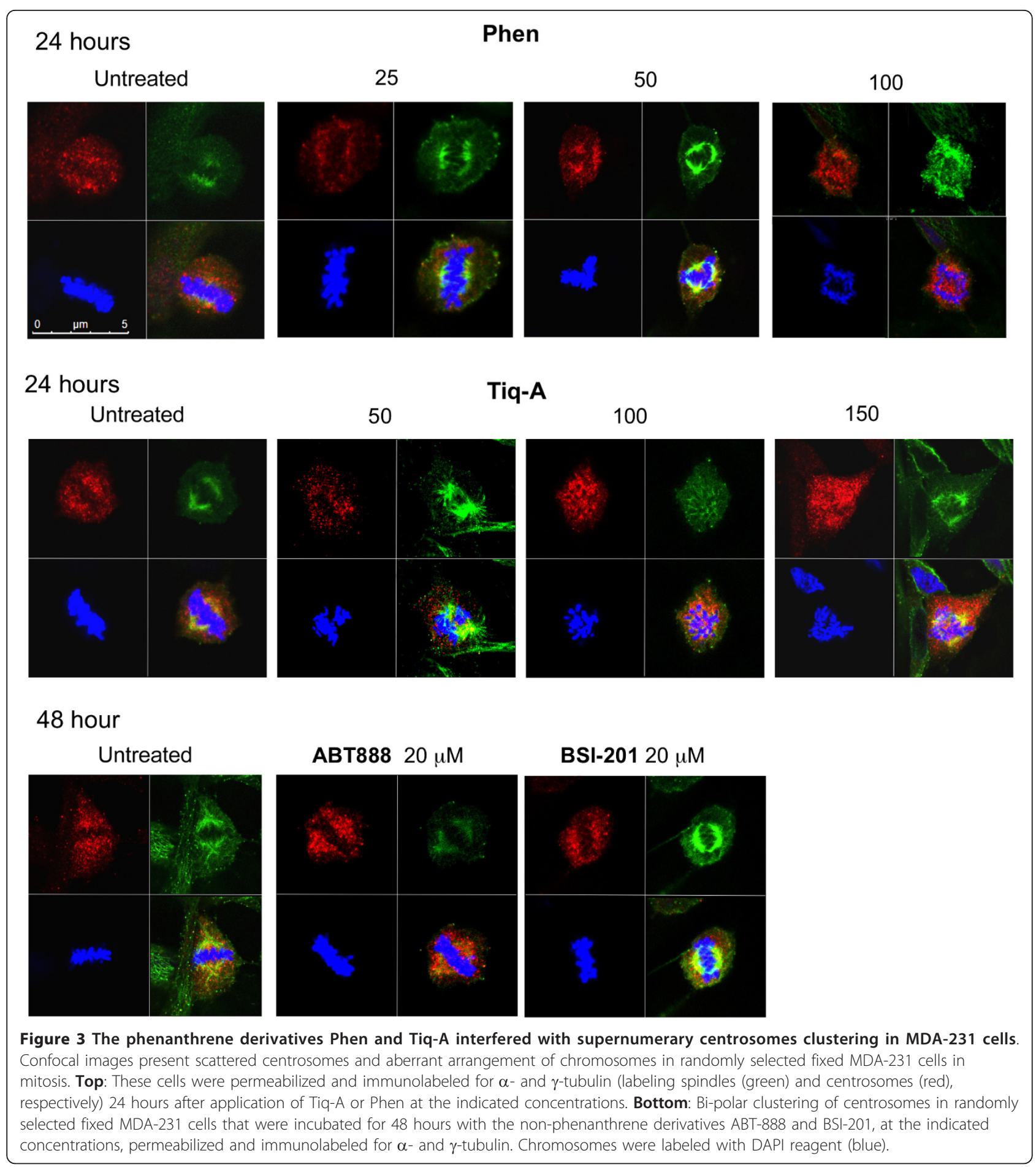

cancer cells target microtubule polymerization (vinca alkaloids, taxanes, colchicines, and also griseofulvin). These compounds therefore impair normal cells as well, and cause severe side effects [36-39].

The discovered potency of PJ-34 in preventing bipolar clustering of extra centrosomes in mitosis may pave the way for a new efficient therapy lacking intolerable side effects, which is based on compounds that exclusively target the unique dependence of many human cancer cells on their supernumerary centrosomes clustering for their survival.

Since many human cancer cells have high occurrence of multicentrosomal cells, the exclusive cytotoxic activity of PJ-34 in multi-centrosomal human cancer cells holds 


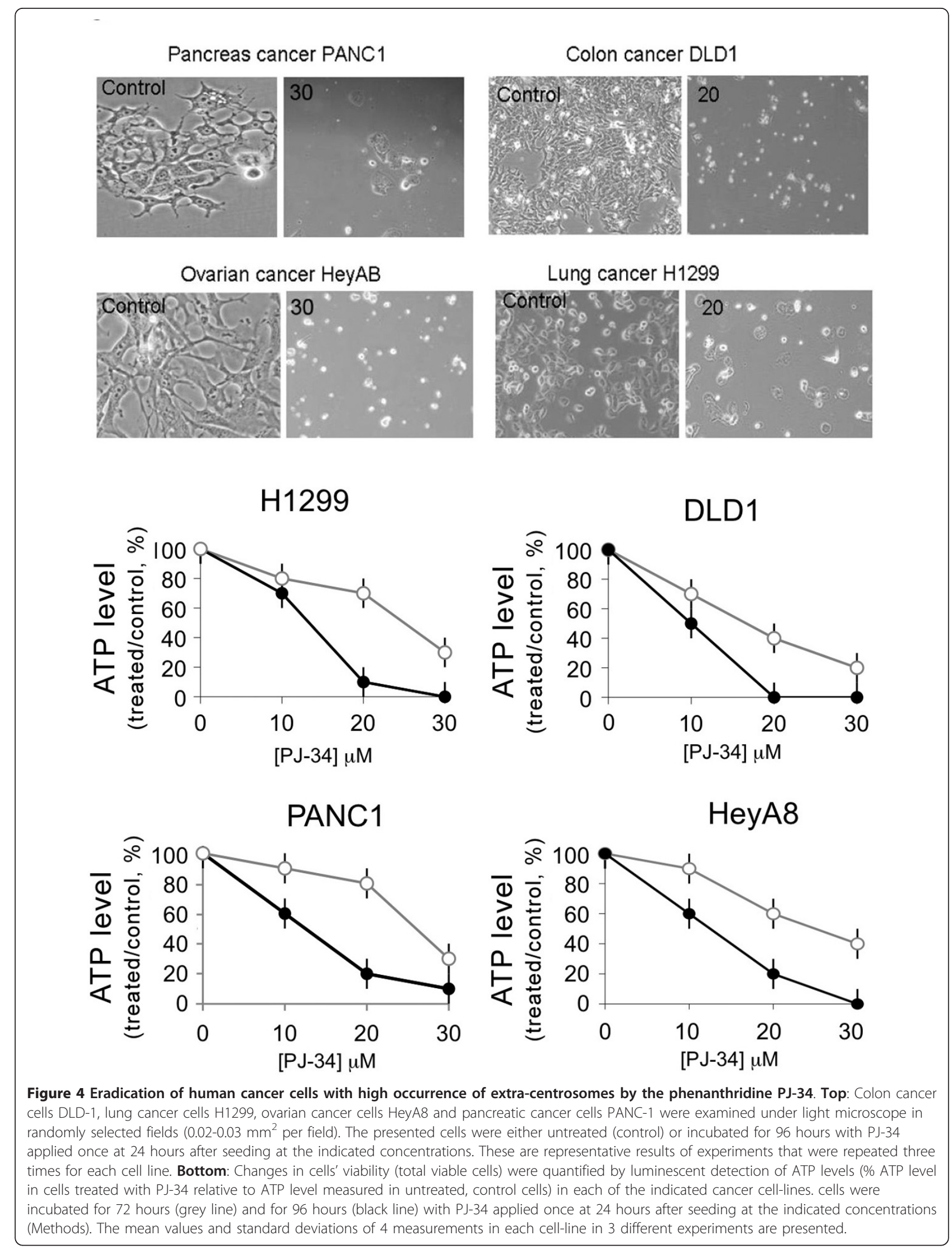




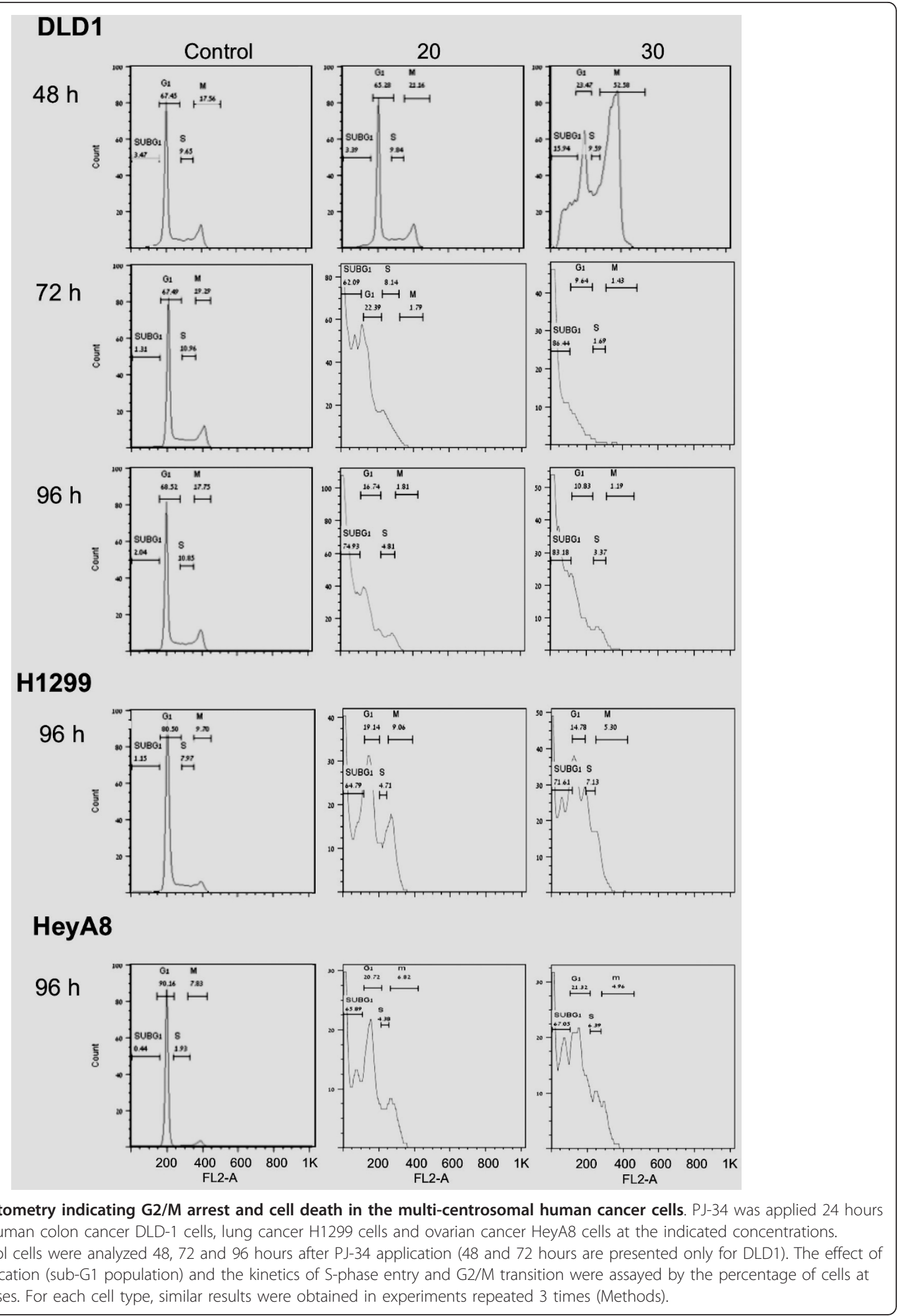




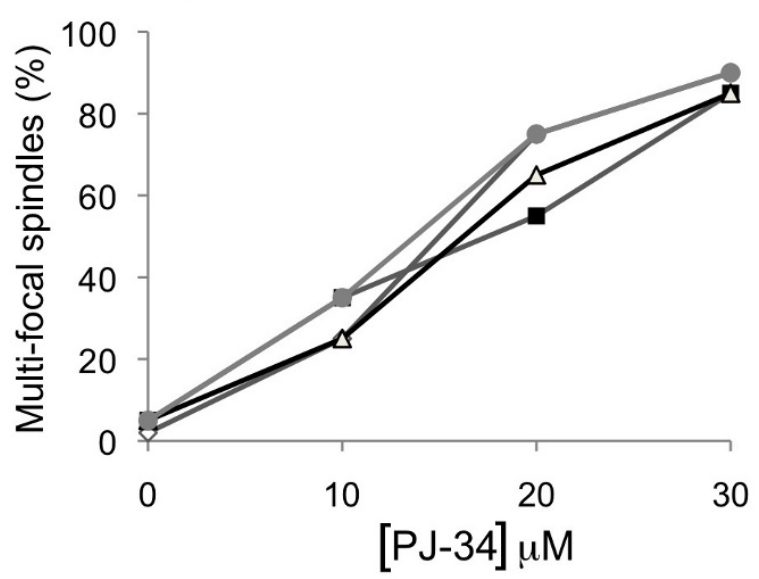

DLD1

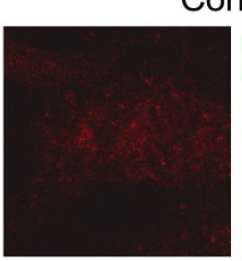

Control
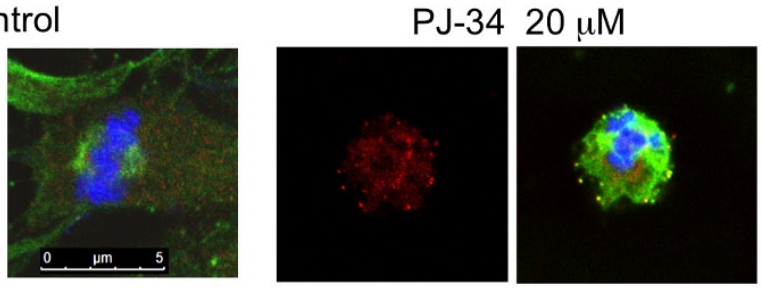

H1299

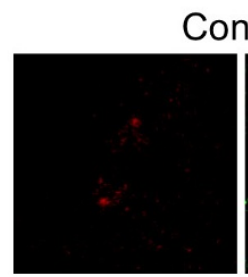

Control

PJ-34 $30 \mu \mathrm{M}$
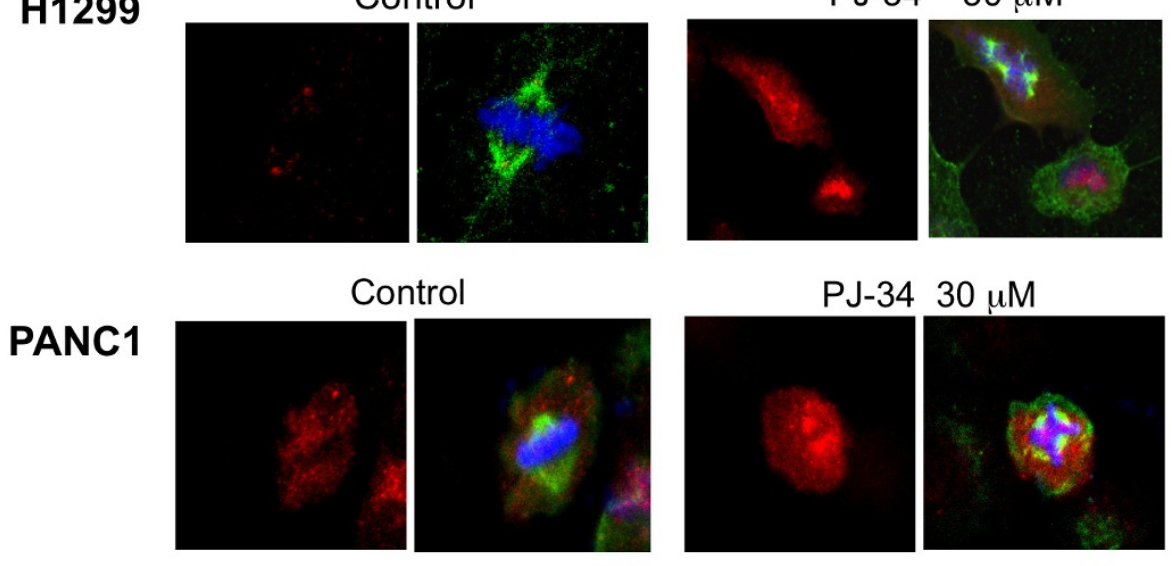

HeyA8

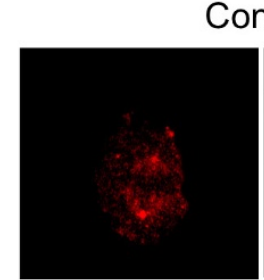

\section{Control}

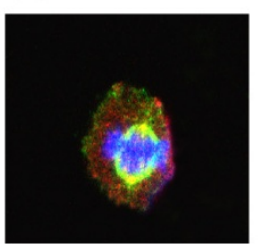

\section{PJ-34 $30 \mu \mathrm{M}$}
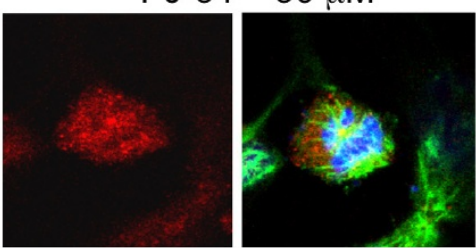

Figure 6 The phenanthridine PJ-34 prevented bi-polar clustering of extra-centrosomes in the tested human cancer cells. Top: PJ-34 interfered with the bifocal clustering of centrosomes in the indicated human cancer cells (DLD1, grey line, solid grey dots; H1299, grey line, empty diamonds; HeyA8, grey line, solid black squares; PANC1, black line, empty triangles) fixed 72 hours after application of PJ-34 at the indicated concentrations. Control and treated cells were permeabilized and immunolabeled for $\alpha$ - and $\gamma$-tubulin, for the detection of spindles (green) and centrosomes (red), respectively, as described below. The percentage of multi-focal spindles was calculated out of 20 spindles detected in randomly selected fixed cells by confocal microscopy in 3 different experiments. Bottom: Confocal images indicate scattered centrosomes and multi-focal spindles in randomly selected fixed cells in mitosis of the indicated cell-lines, 48 hours after application of PJ-34 at the indicated concentrations. Control (untreated) and treated cells were permeabilized and immunolabeled for $\alpha$ - and $\gamma$-tubulin (green and red, respectively). Chromosomes were labeled with DAPI reagent (blue). 

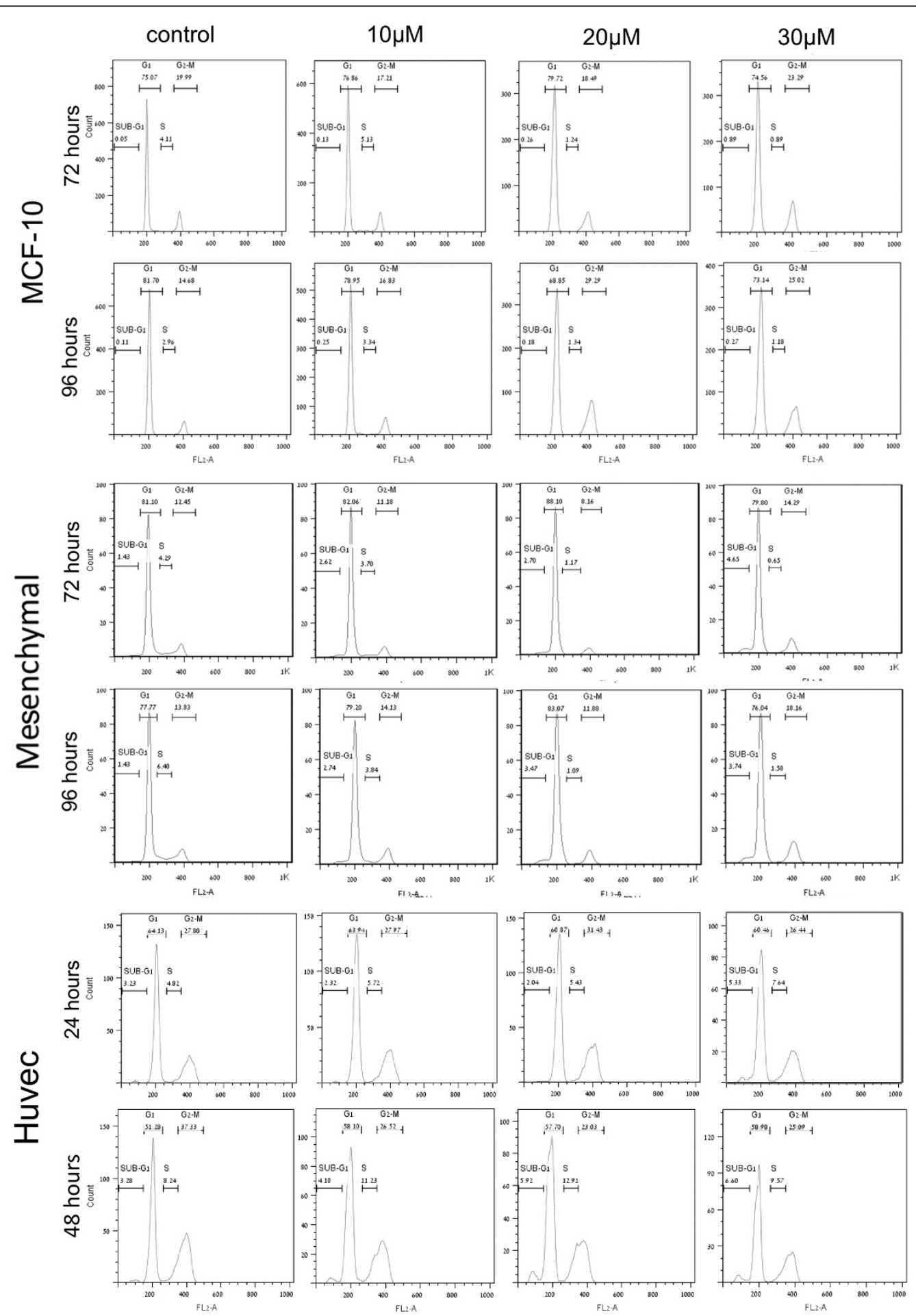

Figure 7 The phenanthridine PJ-34 did not affect the cell-cycle of human normal proliferating cells. Flow cytometry analysis shows that PJ-34 did not affect the cell cycle of human mammary epithelial cells MCF-10, adipose-derived mesenchymal stroma cells prepared from thymus, and human umbilical vein endothelial cells (Huvec). Cells were incubated for the indicated periods with the indicated concentrations of PJ-34 applied 24 hours after seeding. For each cell type, similar results were obtained in experiments repeated 3 times (Methods). 


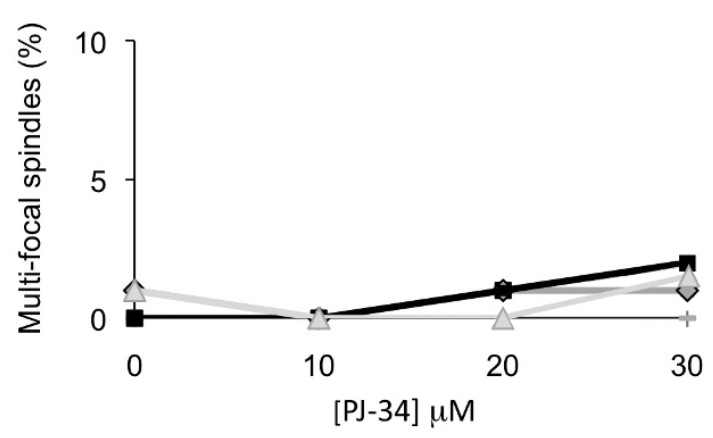

\section{MCF-10}

Control

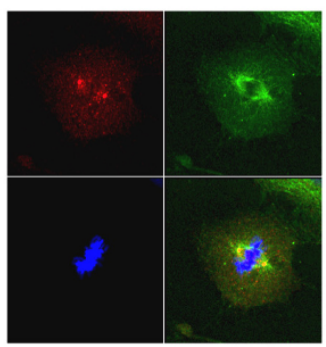

Mesencymal

Control

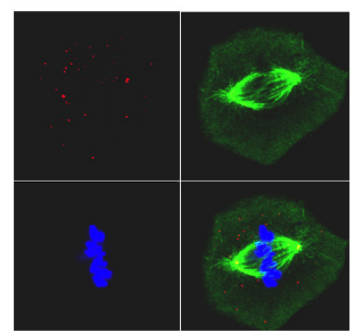

Huvec

Control

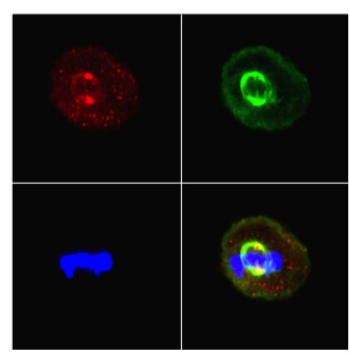

$10 \mu \mathrm{M}$

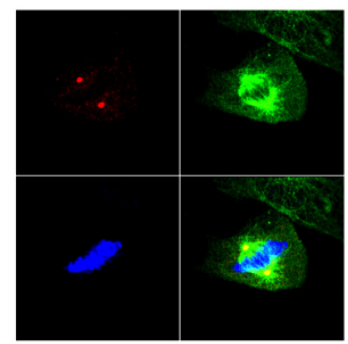

$10 \mu \mathrm{M}$

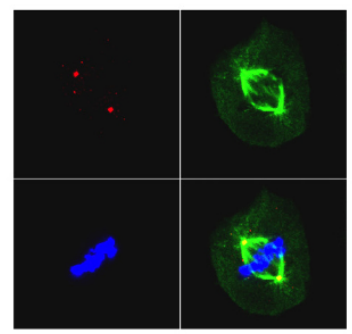

$10 \mu \mathrm{M}$

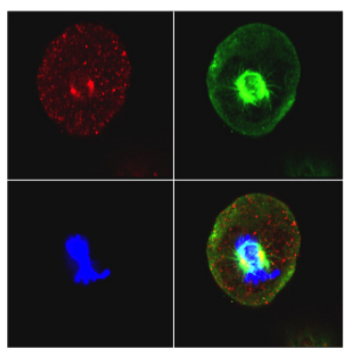

$20 \mu \mathrm{M}$

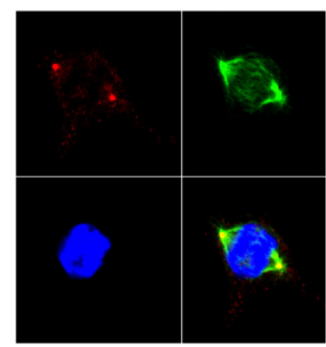

$20 \mu \mathrm{M}$

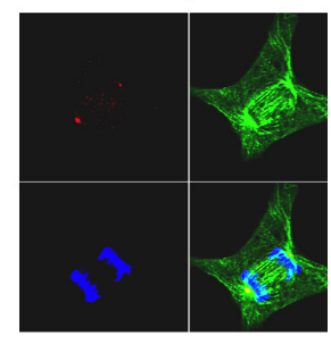

$20 \mu \mathrm{M}$

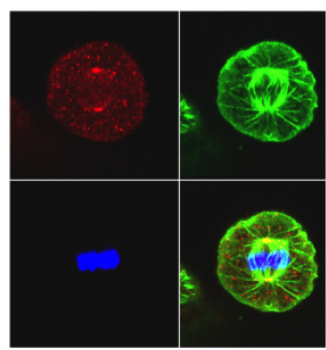

Figure 8 Centrosomes assembly, bifocal spindles and chromosomes segregation were not impaired by PJ-34 in normal human proliferating cells. Epithelial cells MCF-10, mesenchymal stroma cells and endothelial cells (Huvec) were scanned by confocal microscope after 72 hours incubation in the absence or presence of $20 \mu \mathrm{M}$ PJ-34, applied 24 hours after seeding at the indicated concentrations. Top: Percentage of multi-focal spindles (approximately zero) in randomly selected fixed cells was calculated out of at least 20 detected spindles in 3 different experiments performed with each cell type (MCF-10, black line, solid black square; mesenchymal cells, grey line, solid grey diamond; Huvec, grey line, grey triangle). Control (untreated) and treated cells were permeabilized and immunolabeled for $\alpha$ - and $\gamma$-tubulin for the detection of spindles and centrosomes, respectively, as described below. Bottom: Confocal images of randomly selected cells in mitosis, untreated (control) cells, and cells that were incubated with PJ-34 for 72 hours at the indicated concentrations. Untreated cells and cells treated with PJ-34 were permeabilized and immunolabeled for $\alpha$ - and $\gamma$-tubulin (green and red, respectively) for detection of spindles and centrosomes, respectively. Chromosomes were labeled with DAPI reagent (blue). 
promise as a therapeutic tool for a selective chemotherapy in a wide range of human cancers, beyond the currently used selective therapeutic tools targeting specific genes/proteins that affect few cancer cell types [43-45].

\section{Conclusion}

The identification of a molecule exclusively targeting the unique dependence of most human cancer cells on their supernumerary centrosomes bi-polar clustering for their survival, paves the way for a new highly selective cancer-targeting therapy, efficient in a wide range of human cancers.

\section{Additional material}

Additional file 1: Live confocal imaging of MDA-231 cell in mitosis in the presence of the phenanthridine PJ-34. Mitosis with scattered centrosomes ended by cell death in a randomly selected MDA-231 cell that was incubated for 18 hours with PJ-34 $(20 \mu \mathrm{M})$ applied 24 hours after transfection by the indicated vectors, and then scanned over-night (approximately 15 hours) in the presence of PJ-34 by confocal microscope. Cells were transfected by vectors encoding for $\gamma$-tubulin-GFP (for labeling of centrosomes; green) and for histone H2B-RED (for labeling of chromosomes; red).

Additional file 2: Live confocal imaging of MDA-231 cell in interphase in the presence of the phenanthridine PJ-34. Interaction between centrosomes in a randomly selected MDA-231 cell in interphase. This cell was incubated with PJ-34 (20 $\mu \mathrm{M}, 18$ hours incubation) applied 24 hours after transfection by vectors encoding for $\gamma$ tubulin-GFP (green, for labeling of centrosomes) and for $\alpha$-tubulin-RFP (red, for labeling of microtubule arrays in the cytoskeleton) and scanned overnight in the presence of PJ-34 (approximately 15 hours) by confocal microscope.

\section{Acknowledgements}

This work was supported by the Feingrhot Karol and Leonora prize for cancer research (to M.C-A) and by the Israeli Science Foundation (to SI).

\section{Author details}

'The Neufeld Cardiac Research Institute, Department of Physiology and Pharmacology, Sackler Faculty of Medicine, Tel-Aviv University, 69978, TelAviv, Israel. ${ }^{2}$ Department of Human Molecular Genetics and Biochemistry, Sackler Faculty of Medicine, Tel-Aviv University, 69978, Tel-Aviv, Israel. ${ }^{3}$ Imaging Unit, Sackler Faculty of Medicine, Tel-Aviv University, 69978, TelAviv, Israel. ${ }^{4}$ Cancer Research Center, Sheba Medical Center, Tel-Hashomer, 52621 Ramat-Gan, Israel. ${ }^{5}$ Biotechnology and Cell Signaling, UMR7242, Ecole Superieure de Biotechnologie Strasbourg, F-67400, Illkrich-Graffenstaden, France.

\section{Authors' contributions}

LV and AC performed the experiments SI and AC contributed new analytical tools. LM performed the confocal measurements, FD-proofreading of the manuscript. MC-A designed the experiments and wrote the manuscript. All authors read and approved the final manuscript.

\section{Competing interests}

M. Cohen-Armon is the inventor of patent WO 2009/0477052, owned by the Tel-Aviv University. The co-authors declare that they have no competing interests. This study was not supported by any company or commercial fund.

Received: 20 May 2011 Accepted: 26 September 2011 Published: 26 September 2011
References

1. Inbar-Rozensal D, Castiel A, Visochek L, Kastel D, Dantzer F, Izraeli S, CohenArmon M: A selective eradication of human nonhereditary breast cancer cells by phenanthridine-derived polyADP-ribose polymerase inhibitors. Breast Canc Res 2009, 11/6/R78:1-11.

2. Chiarugi A, Meli E, Calvani M, Picca R, Picca R, Baronti R, Camaioni E, et al: Novel isoquinolinone-derived inhibitors of polyADP-ribose polymerase-1: Pharmacological characterization and neuroprotective effects in an invitro model of cerebral ischemia. J Pharmacol Exp Ther 2003, 305:943-949.

3. Jagtap P, Szabo C: Poly(ADP-ribose)polymerase and the therapeutic effects of its inhibitors. Nat Rev Drug Discovery 2005, 4:421-440.

4. Gergely F, Basto R: Multiple centrosomes: together they stand, divided they fall. Genes Dev 2008, 22:2291-2296.

5. Godinho SA, Kwon M, Pellman D: Centrosomes and cancer: how cancer cells divide with too many cetrosomes. Canc Met Rev 2009, 28:85-98.

6. Doxsey S: Re-evaluating centrosome function. Nat Rev Mol Cell Biol 2001, 2:688-698

7. Walczak CE, Heald R: Mechanisms of mitotic spindle assembly and function. In International Rev of Cytology. Volume 265. (Elsevier Inc.); 2008:111-158

8. Bettencourt-Dias M, Glover D: Centrosome biogenesis and function: centrosomics brings new understandings. Nat Rev Mol Cell Biol 2007 8(6):451-463

9. Cunha-Ferreira I, Bento I, Bettencourt-Dias M: From zero to many: control of centriole number in development and disease. Traffic 2009, 10:482-498.

10. Galimberti F, Thompson SL, Ravi S, Compton D, Dmitrovsky E: Anaphase Catastrophe Is a Target for Cancer Therapy. Clin Cancer Res 2011, 17:1218-1222.

11. Tsou M-FB, Stearns T: Controlling centrosome number: licenses and blocks. Cur Opin Cell Biol 2006, 18:74-78.

12. Leber B, Maier B, Fuchs F, Chi J, Riffel P, Anderhub S, Wagner L, Ho AD, Salisbury JL, Boutros M, Krämer A: Proteins Required for Centrosome Clustering in Cancer Cells. Sci Transl Med 2010, 2(33).

13. Cohen-Armon M, Visochek L, Rozensal D, Kalal A, Geistrikh I, Klein R, Bendetz-Nezer S, Yao Z, Seger R: DNA-independent PARP-1 activation by phosphorylated ERK2 increases Elk1 activity: a link to histone acetylation. Mol Cell 2007, 25:297-308.

14. Stearns $T$, Evans $L$, Kirschner M: $Y$-Tubulin is a highly conserved component of the centrosome. Cell 1991, 65:825-836.

15. Vayssiere $J \mathrm{~L}$, Petit $\mathrm{PX}$, Risler $\mathrm{Y}$, Mignotte $\mathrm{B}$ : Commitment to apoptosis is associated with changes in mitochondrial biogenesis and activity in cell lines conditionally immortalized with simian virus 40. Proc Natl Acad Sci USA 1994, 91:11752-11756.

16. Rieder CL, Maiato H: Stuck in Division or Passing through: What Happens When Cells Cannot Satisfy the Spindle Assembly Checkpoint. Dev Cell 2004, 7:637-651.

17. Kwon M, Godinho SA, Chandhok NS, Ganem NJ, Azioune A, Thery M, Pellman D: Mechanisms to suppress multipolar divisions in cancer cells with extra centrosomes. Genes Dev 2008, 22:2189-2203.

18. Rouleau M, Patel A, Hendze MJ, Kaufmann SH, Poirier GG: PARP inhibition: PARP1 and beyond. Nature Rev Cancer 2010, 10:293-301.

19. Krishnakumar R, Kraus WL: The PARP side of the nucleus: Molecular actions, physiological outcomes, and clinical targets. Mol Cell 2010, 39:8-24.

20. Chang W, Dynek JN, Smith S: NuMA is a major acceptor of poly(ADPribosyl)ation by tankyrase 1 in mitosis. Biochem J 2005, 391:177-184.

21. Chang P, Jacobson MK, Mitchison TJ: PolyADP-ribose is required for spindle assembly and structure. Nature 2004, 432:645-649.

22. Earl E, Saxena A, MacDonald A, Hudson DF, Shaffer LG, et al: PolyADPribose polymers at active centromers and neocentromers at metaphase. Hum Mol Gen 2000, 9:187-194.

23. Saxena A, Saffery R, Wong LH, Kalitsis P, Choo A: Centromere Proteins Cenpa, Cenpb, and Bub3 Interact with Poly(ADP-ribose) Polymerase-1 Protein and Are Poly(ADP-ribosyl)ated. J Biol Chem 2002, 277:26921-26926.

24. Kanai M, Tong WM, Sugihara E, Wang ZQ, Fukasawa K, Miwa1 M: Involvement of Poly(ADP-Ribose) Polymerase 1 and Poly(ADP-Ribosyl) ation in Regulation of Centrosome function. Mol Cell Biol 2003, 23:2451-2462.

25. Ogino H, Nozaki T, Gunji A, Maeda M, Suzuki H, Ohta T, Murakami Y, Nakagama H, Sugimura T, Masutani M: Loss of Parp-1 affects gene 
expression profile in a genome-wide manner in ES cells and liver cells. BMC Genomics 2007, 8:41

26. Coombs MM, Bhatt TS, Croft CJ: Correlation between carcinogenicity and chemical structure in Cyclopenta[a]- phenanthridines. Canc Res 1973, 33:832-837.

27. Phillips SD, Castle RN: A review of the chemistry of the antitumor benzo[c]phenanthridine alkaloids nitidine and fagaronine and of the related antitumor alkaloid coralyne. Journal of Heterocyclic Chemistry 1981, 18:223-232.

28. Turchi G, Glatt HR, Seidel A, Puliti A, Sbrana I: Structure activity relationship in the induction of chromosomal aberrations and spindle disturbances in Chinese hamster epithelial liver cells by regioisomeric phenanthridine quinines. Cell Biology and Toxicology 1997, 13:155-165.

29. Lamoral-Theys D, Andolfi A, Van Goietsenoven G, Cimmino A, Le Calve B, Wauthoz N: Lycorine, the main phenanthridine Amaryllidaceae alkaloid, exhibits significant antitumor activity in cancer cells that display resistance to proapoptotic stimuli: an investigation of structure-activity relationship and mechanistic insight. J Med Chem 2009, 52:6244-6256.

30. Iwasaki H, Okabe T, Takara K, Toda T, Shimatani M, Oku H: Tumor-selective cytotoxicity of benzo[c] phenanthridine derivatives from Toddalia asiatica Lam. Cancer Chemother Pharmacol 2010, 65:719-726.

31. Ingle WL, Salisbury JL: The role of the centrosomes in the development of malignant tumors. Current topics in Dev Biol 1999, 49:313-329.

32. Ganem NJ, Godunov SA, Pellman D: A mechanism linking extra centrosomes to chromosomal instability. Nature 2009, 460:278-282.

33. Fukasaw K: Oncogenes and tumour suppressors take in centrosomes. Nature Rev/Cancer 2007, 7:911-924.

34. Fielding AB, Lim S, Montgomery K, Dobreva I, Dedhar S: critical role of integrin-linked kinase, ch-TOG and TACC 3 in centrosome clustering in cancer cells. Oncogene 2011, 30:521-534.

35. Galimberti F, Thompson SL, Liu X, Li H, Memoli V, et al: Targeting the Cyclin E-Cdk-2 Complex Represses Lung Cancer Growth by Triggering Anaphase Catastrophe. Clin Canc Res 2010, 16:109-120.

36. Jordan MA, Wilson L: Microtubules as a target for anticancer drugs. Nat Rev Cancer 2004, 4:253-265.

37. Chen JG, Horwitz SB: Differential mitotic responses to microtubulestabilizing and de-stabilizing drugs. Cancer Res 2002, 62:1935-1938.

38. Rebacz $\mathrm{B}$, Larsen $\mathrm{TO}$, Clausen $\mathrm{MH}$, Rønnest $\mathrm{MH}$, Löffler $\mathrm{H}$, Ho AD, Krämer $\mathrm{A}$ : Identification of Griseofulvin as an inhibitor of centrosomal clustering in a phenotype-based screen. Canc Res 2007, 67:6342-6350.

39. Jackson JR, Denis R, Patrick DR, Mohammed M, Dar MM, Huang PS: Targeted anti-mitotic therapies: can we improve on tubulin agents? Nature Rev Cancer 2007, 7:107-117.

40. Kozielski F, DeBonis S, Skoufias DA: Screening for inhibitors of microtubule-associated motor proteins. Methods Mol Med 2007, 137:189-207.

41. Manning JA, Kuma S: A potential role for NEDD1 and the centrosome in senescence of mouse embryonic fibroblasts. Cell Death Dis 2010, 1:e35.

42. Riffell JL, Janicke RU, Roberge M: Caspase-3-Dependent Mitotic Checkpoint Inactivation by the Small-Molecule Inducers of Mitotic Slippage SU6656 and Geraldol. Mol Cancer Ther 2011, 10:839-849.

43. Davies H, Bignell GR, Cox C, Stephens P, Edkins S, Clegg S, Teague J, et al: Mutations of the B-RAF gene in human cancer. Nature 2002, 417:949-954.

44. Bild AH, Potti A, Nevins JR: Linking oncogenic pathways with therapeutic opportunities. Nat Rev Cancer 2006, 6:734-741.

45. O'Connor MJ, Martin NM, Smith GC: Targeted cancer therapies based on the inhibition of DNA strand break repair. Oncogene 2007, 26:7816-7824.

\section{Pre-publication history}

The pre-publication history for this paper can be accessed here: http://www.biomedcentral.com/1471-2407/11/412/prepub

doi:10.1186/1471-2407-11-412

Cite this article as: Castiel et al: A phenanthrene derived PARP inhibitor is an extra-centrosomes de-clustering agent exclusively eradicating human cancer cells. BMC Cancer 2011 11:412.

\section{Submit your next manuscript to BioMed Central and take full advantage of:}

- Convenient online submission

- Thorough peer review

- No space constraints or color figure charges

- Immediate publication on acceptance

- Inclusion in PubMed, CAS, Scopus and Google Scholar

- Research which is freely available for redistribution

Submit your manuscript at www.biomedcentral.com/submit 